\title{
Velocity fluctuations of initially stratified sedimenting spheres
}

\author{
Shang-You Tee \\ Department of Physics and Division of Engineering and Applied Sciences, Harvard University, \\ Cambridge, Massachusetts 02138, USA \\ P. J. Mucha \\ Department of Mathematics and Institute for Advanced Materials, University of North Carolina, \\ Chapel Hill, North Carolina 27599, USA \\ M. P. Brenner \\ Division of Engineering and Applied Sciences, Harvard University, Cambridge, Massachusetts 02138, USA \\ D. A. Weitz \\ Department of Physics and Division of Engineering and Applied Sciences, Harvard University, \\ Cambridge, Massachusetts 02138, USA
}

(Received 24 April 2007; accepted 4 October 2007; published online 12 November 2007)

\begin{abstract}
The study of velocity fluctuations in the sedimentation of spheres is complicated by the time evolution of the underlying particle distribution, both at the microscale and in the bulk. We perform a series of experiments and simulations to isolate the effect of an initial, stable stratification in the particle concentration. The directly observed dependence of velocity fluctuations on stratification agrees with a previously obtained scaling theory. (C) 2007 American Institute of Physics.
\end{abstract}

[DOI: $10.1063 / 1.2806597]$

Sedimentation is a fundamental fluids process of broad importance that possesses interesting physics and perplexing behaviors. Even the seemingly simplest situation, the slow sedimentation of a dilute collection of monodisperse spheres in a Newtonian fluid, continues to generate questions of intriguing difficulty. While the mean sedimentation velocity is well understood, ${ }^{1,2}$ the velocity fluctuations about this mean velocity are more complicated. Caflisch and Luke found that the long-ranged hydrodynamic nature of the interactions can cause the magnitude of velocity fluctuations to diverge with system size. ${ }^{3}$ A number of experiments, however, have concluded that fluctuations are independent of system size. ${ }^{4-7}$ Recent investigations have identified the importance of stratification of the sedimenting particle density, ${ }^{8}$ and pinpointed the changing stratification as playing an important role in the decay of velocity fluctuations over time, ${ }^{9}$ as the stratification changes continuously in time due to the spreading front ${ }^{10}$ and particle polydispersity. ${ }^{11}$ Such results have led to a model wherein locally steady fluctuations can be controlled by stratifications of the particle density above a very small critical stratification, in apparent agreement with experiments and simulations, ${ }^{12}$ though full model verification is complicated by the time evolution of the particle distributions and fluctuations. Meanwhile, new experiments ${ }^{11}$ and simulations ${ }^{13}$ have demonstrated situations in which stratification does not appear to be the controlling mechanism of nonsystem-size-scaling fluctuations, while similar stratification effects have been shown to play a role in wavelength selection in the instability of sedimenting spheroids. ${ }^{14}$ Amid such uncertainties, a more controlled investigation is warranted to quantify the dependence of velocity fluctuations on stratification in situations where stratification is the dominant controlling mechanism.

In this article, we demonstrate through experiments and simulations that the velocity fluctuations decrease with increasing stratification, in quantitative agreement with an existing scaling model. ${ }^{9,10,12}$ To avoid complications from evolving density profiles, we choose a series of experiments and simulations initialized with dilute, linear particle concentration profiles, at physical parameters minimizing mixing so the initial profile is well-maintained in time, up to the nearconstant average downward transport. Because of the absence of appreciable time evolution, we can effectively isolate and quantify the dependence of velocity fluctuations on stratification.

Velocity fluctuations are driven by particle density fluctuations, as demonstrated by a physical argument due to Hinch. ${ }^{15}$ assuming that a vertically homogeneous dilute random sample obeys Poisson statistics, a region of size $\ell$ with particles of radius $a$ at volume fraction $\phi$ has a typical concentration fluctuation $\Delta \phi \sim \sqrt{\phi a^{3} / \ell^{3}}$. The velocity of such a region is determined by balancing its buoyant weight, $(\Delta \phi) \ell^{3} \Delta \rho g \sim(\Delta \phi) \ell^{3}\left(\eta V_{0} / a^{2}\right)$, with its Stokes drag, $\sim \eta \ell \Delta V$, giving $\Delta V_{\ell}=C V_{0} \sqrt{\phi \ell / a}$, with $V_{0}$ the Stokes velocity of an isolated particle. The dominant $\Delta V$ in homogeneous sedimentation is then set by the smallest container dimension, $d$, because the above arguments break down for $\ell>d$, giving

$$
\Delta V_{\text {Poisson }}=C V_{0} \sqrt{\phi d / a}
$$

in agreement with Caflisch and Luke. ${ }^{3}$ These fluctuations lead to long-time diffusive mean squared displacements with effective diffusivity, ${ }^{16,10} D \sim \ell \Delta V$. Density fluctuations on a given length scale are then produced and destroyed due to randomness on the same time scale, $\tau_{D} \approx \ell^{2} / D \approx \ell / \Delta V$, advecting a distance $\sim \ell$ in their lifetime. The competition between fluctuation creation and destruction drives almost any 
vertically homogeneous system to Poisson statistics at long length scales, consistent with the assumed statistically steady density fluctuations.

Even a very small stable particle density stratification modifies the above argument. The buoyancy mismatch of density fluctuations is lost if the $\Delta \phi$ density fluctuations are smaller than the stratification concentration change over a swirl lifetime translating distance $\sim \ell$, meaning such fluctuations can no longer advect as far as in the vertically homogeneous case. For a locally linear decrease in $\phi$ in the direction opposite to the average motion, $\phi=\phi_{0}(1-\beta z)$, with $\beta>0$, the stratification suppresses fluctuations for scales bigger than those where the two changes in concentration balance, $\beta \phi_{0} \ell \sim \Delta \phi$, that is, $\ell \sim a \phi^{-1 / 5}(\beta a)^{-2 / 5}$, with velocities $\Delta V \sim V_{0} \phi^{2 / 5}(\beta a)^{-1 / 5}$. These arguments for stratification control of fluctuations apply only when $\ell<d$; otherwise, in the absence of other controlling mechanisms, the fluctuations are set by the container size $d$. The same scalings are obtained from analysis of a model stochastic continuum equation. ${ }^{12}$ Setting $\ell \sim d$, we define the critical stratification,

$$
\beta_{\text {crit }} \equiv a^{3 / 2} d^{-5 / 2} \phi^{-1 / 2}=\left(d \sqrt{N_{d}}\right)^{-1},
$$

where $N_{d}$ is the number of particles in a volume of radius $d$, with $\Delta V^{2} / \Delta V_{\text {Poisson }}^{2} \sim\left(\beta / \beta_{\text {crit }}\right)^{-2 / 5}$ for $\beta$ above the critical stratification. This critical stratification decreases rapidly as the cell thickness $d$ increases, with the actual crossover at an unknown but presumably $O(1)$ scalar multiple of the $\beta_{\text {crit }}$ defined in Eq. (2). Earlier studies ${ }^{10,12}$ indicated this crossover to be near $\frac{1}{2} \beta_{\text {crit }}$, though the crossover was not systematically studied before.

In our experiments, polystyrene beads of radius $a=14.3 \pm 0.1 \mu \mathrm{m}$ and volume fractions $\phi$ of $0.01 \%$ and $0.1 \%$ are suspended in sucrose solution in a $d \times w \times h$ cell with horizontal dimensions $d=2 \mathrm{~cm}$ and $w=3 \mathrm{~cm}$, and height $h=30 \mathrm{~cm}$. The viscometer-measured viscosities of the sucrose solutions range from $\eta=1$ to $10 \mathrm{cP}$ with varying sugar concentration. The cell is immersed in a stirred water bath at a temperature $22.0 \pm 0.1{ }^{\circ} \mathrm{C}$. The nearly uniform sizes of the particles limit particle concentration gradients due to polydisperse effects. Likewise the small cell dimension is chosen to limit bulk transport due to internal mixing and hydrodynamic diffusion of the front. Low Reynolds numbers $\left(\operatorname{Re} \sim 10^{-3}\right)$ and high Peclet numbers $\left(\mathrm{Pe} \sim 10^{7}\right)$ indicate that inertia and Brownian motion can be neglected. Since the density of the sucrose solutions is higher than that of the particles, the mean velocity of the particles in these experiments is always upwards to the top of the cell. Velocity fluctuations are measured by particle image velocimetry (PIV) with a CCD camera imaging an area $11.3 \times 8.5 \mathrm{~mm}$, with $5 \mathrm{~mm}$ depth of focus, $\approx h / 4$ away from the top boundary. We determine the vertical velocity fluctuations by $\Delta V=\langle(V$ $\left.-\langle V\rangle)^{2}\right\rangle^{1 / 2}$, where $V$ is the local vertical velocity component and $\langle V\rangle$ is the mean velocity in the window.

A linear stratification is established using the Oster double bucket technique. ${ }^{17}$ The first bucket is filled with dense sucrose solution with a known volume fraction of particles while the second bucket is filled with a higher volume fraction of particles in a less dense sucrose solution. The

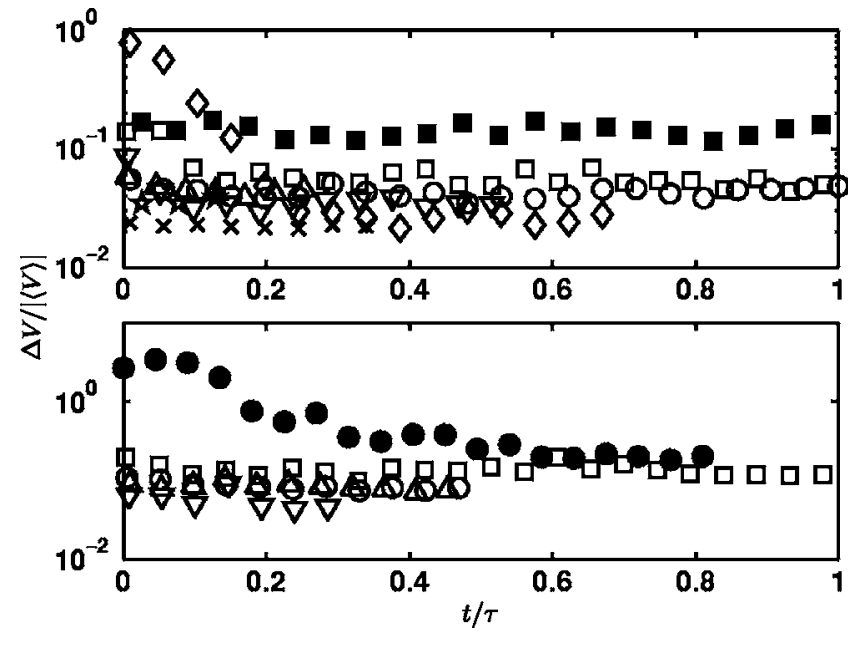

FIG. 1. PIV-measured vertical velocity fluctuations vs time $(t / \tau$, where $\tau=h /|\langle V\rangle|$ and $|\langle V\rangle|$ is the average settling velocity in the imaged area). The average volume fractions are $\phi=0.01 \%$ (top half) and $\phi=0.1 \%$ (bottom half). Velocity fluctuations in the absence of initially imposed stratification are plotted in filled symbols. Measured fluctuations are presented up until times when fluctuations drop off rapidly as the sediment front approaches the imaged area. Because the average velocity varies appreciably across the cell with varying sucrose density, experiments with larger sucrose gradients (typically those with smaller particle concentration gradients) can therefore include data up until $t / \tau \approx 1$. At $\phi=0.01 \%$ (top half), the initially imposed stratifications, quantified by $\beta / \beta_{\text {crit }}$ (as defined in the text) are $0(\mathbf{\square}), 4.2$ $(\square), 8.8(\bigcirc), 16.6(\triangle), 28.4(\nabla), 59(\diamond), 105(\times)$, and $427(\star)$. At $\phi=0.1 \%$ (bottom half), the initially imposed stratifications are $0(\bullet), 8.2$ ( $\square), 46(\bigcirc), 92(\triangle)$, and $339(\nabla)$.

suspension from the first bucket is stirred continuously and passed through a flow diffuser onto the existing fluid surface in the cell; simultaneously the suspension from the second bucket is allowed to flow into the first bucket. By keeping these two flow rates equal, a stable linear particle concentration profile is created with larger (smaller) volume fraction at the top (bottom) of the cell. A side effect is that a stable sucrose density gradient is also established; but the sucrose density difference in the imaged area varies less than 5\% across all runs and therefore should not appreciably affect the fluctuation measurements here. In principle, no sucrose variation is strictly necessary, but we found it difficult to establish small stable particle concentration stratifications without an accompanying sucrose stratification, the differences in suspension density due to the particle concentration stratification alone insufficient to prevent mixing near the flow diffuser in our setup.

Local initial volume fractions at selected heights are determined by measuring the transmittance of a laser beam through the cell with a photodiode and comparing against known transmittance-volume fraction calibration curves. This measurement is further augmented by pipetting small amounts of fluid from different heights of the cell after the experiment and weighing with a scale balance. Assuming complete mixing in the first bucket yields volume fractions and densities in good agreement with the transmittance measurements and with the measured weights of the pipetted volumes.

The resulting velocity fluctuations with time are plotted in Fig. 1. For a $\phi=0.01 \%$ suspension that is shaken carefully 


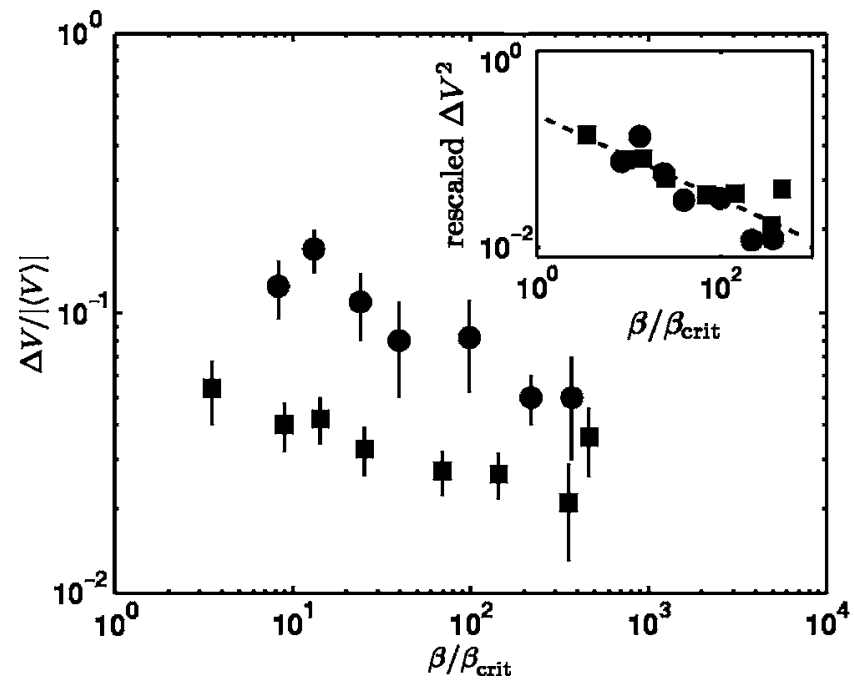

FIG. 2. Time-averaged vertical velocity fluctuations vs stratification expressed as $\beta / \beta_{\text {crit }}$. Each data point is generated from a single experiment, with volume fractions $\phi=0.01 \%$ ( $)$ and $\phi=0.1 \%(\bullet)$, and error bars indicating the deviation of the fluctuation over the averaging window. The inset figure replots the same $\Delta V^{2}$, rescaled by the corresponding Poisson prediction. The dashed line in the inset is a fit to a power law, yielding an exponent $-0.43 \pm 0.07$.

and checked by transmittance and pipetting to verify there is no stratification, the velocity fluctuations are measured to be steady for the duration of the experiment. When stable linear concentration gradients are created, $\phi=\phi_{0}(1-\beta z)$, the fluctuations each achieve steady values (sometimes after an initial transient), but have smaller magnitudes. The experiment at $\beta / \beta_{\text {crit }}=59$ appears to decay over a longer time scale, but still appears to reach a steady value that is then maintained until the front gets close to the imaged measurement area (not shown in the figure). In each of these cases, the velocity fluctuations and polydispersities are so small that the sedimentation front does not appreciably spread during the experiments, thus ensuring that the initial concentration gradients persist over time. When the volume fraction is increased to $\phi=0.1 \%$, the nonstratified suspension has fluctuations that decay for most of the duration of the experiment, in agreement with previous measurements, ${ }^{9}$ the difference between the $0.01 \%$ and $0.1 \%$ behaviors also being described by the convective model of, e.g., Refs. 7, 13, and 15. However, when a steep volume fraction gradient is initially imposed, the fluctuations for $\phi=0.1 \%$ are both suppressed and relatively steady in time. As steeper stratifications are introduced, the fluctuations are suppressed further. We emphasize that each symbol notating the velocity fluctuations plotted in Fig. 1 corresponds to a single experiment, because of the inherent difficulty in setting up the particle concentration gradient (cf. repeatedly stirring and observing the same suspension). Our data thus includes outliers such as the early times of the diamond symbols in the top half of the figure; nevertheless, we see that even that experiment appears to settle down to statistically steady fluctuations.

Figure 2 shows the time-averaged velocity fluctuations from the experiments versus the initially imposed stratification (ignoring data from the initial transients, and including additional experiments beyond those in Fig. 1). In the main figure, the measured $\Delta V /|\langle V\rangle|$ are plotted, and there is unsurprising separation between the data at $\phi=0.01 \%$ and $\phi=0.1 \%$, because the Poisson-level fluctuations (1) are larger at higher volume fraction. Because the unstratified experiment at $\phi=0.01 \%$ yields relatively constant fluctuations, we use that experiment to determine the constant in Eq. (1), giving $C \doteq 0.39$, which is of the same order as the scalar premultipliers for square-based cells. ${ }^{12}$ The vertical velocity variances for both concentrations collapse when rescaled according to the Poisson prediction (with this $C$ ), as shown in the inset. A power-law fit to the $\Delta V^{2}$ in the Fig. 2 inset indicates an exponent $-0.43 \pm 0.07$, in good agreement with the $-2 / 5$ model prediction.

To further test these results, we performed simulations of sedimenting point particles interacting by wall-modified dilute-limit hydrodynamics, using the technique described in Ref. 12. Each simulation conducted for this study consists of between $10^{5}$ and $2 \cdot 10^{5}$ point particles of hydrodynamic particle radius $a$ settling in a $d \times w \times h$ cell, with no-slip boundary conditions along the parallel side walls (separated by $d=150 a$ or $200 a$ ), periodic boundary conditions in the long horizontal dimension (with $w=4 d$ or $8 d$ ), an impenetrable container bottom and top imposed by simple images (separated by $h=8 d$ ), and hydrodynamic truncations of Ref. 12 of $N_{k}=31$ or 63 . No hindered settling was included here, nor was the effect of the experimental sucrose density gradients modeled in any way. Various linear particle concentration stratifications along the vertical direction were initialized with particle concentrations at the top of the cell between 0 and $100 \%$ of the bottom concentration. In all, 112 individual simulations were computed for initially stratified conditions representing 14 different $\beta / \beta_{\text {crit }}$ values. For comparison, an additional 29 simulations at similar physical parameters were conducted with no initially imposed stratification.

Velocity fluctuations were measured in a window $2-3.2 d$ from the container bottom, that is, from 0.25 to $0.4 h$. The velocity fluctuations in the simulations behaved similarly to the experimental measurements in Fig. 1, except that the variation with time is larger in the simulations here because of the smaller number of particles; for comparison, the experiments here contain $O\left(3 \cdot 10^{5}-10^{7}\right)$ particles. We define the initial concentration at the middle height of the cell to be the reference $\phi_{0}$, and the total sedimentation time $\tau=h /|\langle V\rangle|$ (whereas $|\langle V\rangle|$ varies with the sucrose density gradient in the experiments, it is constant across the height of the simulated cells). We time-average between when the mean settling velocity transports that concentration to the top of the measurement window $(t=0.1 \tau)$ and when this mean transport reaches the bottom window $(t=0.25 \tau)$, with the resulting time-averaged velocity variances in each direction for each simulation plotted versus stratification in Fig. 3. These velocity variances have been rescaled by the no-freeparameters Poisson prediction for each simulation geometry, ${ }^{12}$ consistent with the predicted transition from $\Delta V^{2} \approx \Delta V_{\text {Poisson }}^{2}$ for $\beta \leqslant \beta_{\text {crit }}$ to $\Delta V^{2} / \Delta V_{\text {Poisson }}^{2} \sim\left(\beta / \beta_{\text {crit }}\right)^{-2 / 5}$ for $\beta \gtrsim \beta_{\text {crit }}$.

In Fig. 4, we combine the experimental data with the 


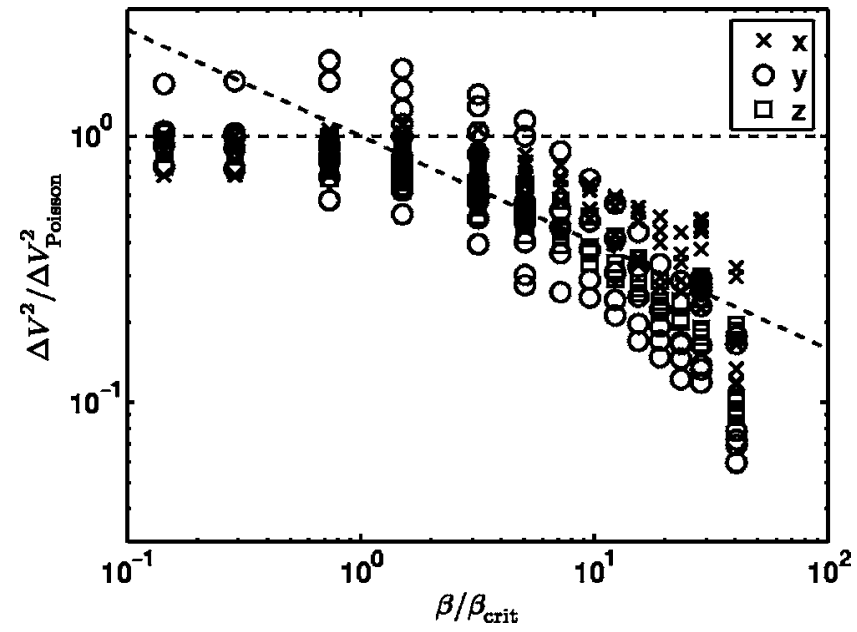

FIG. 3. Time-averaged velocity variances (rescaled by the Poisson prediction) vs stratification, as observed in simulations. Each simulation run yields 3 data points, for velocity fluctuations in the short, wall-bounded direction $(\times)$, the long horizontal direction $(\bigcirc)$, and the vertical direction $(\square)$. The angled dashed line identifies the model-indicated exponent $-2 / 5$, with crossover stratification at $\beta_{\text {crit }}$.

vertical velocity fluctuations from the simulations, the latter now averaged over 5 simulation runs at identical physical parameters. Interestingly, while the experiments and simulations are each separately in reasonable agreement with the theoretical $-2 / 5$ scaling, the experimental and computational results do not lie on the same power-law fit, the experimental data instead shifted to smaller fluctuations at the same $\beta / \beta_{\text {crit. }}$. The data indicates that the experiments crossover to stratification control of the fluctuations at stratifications $\sim 32$ times smaller than in the simulations. That is, comparing the dashed lines in Fig. 4, the line close to the stratificationcontrolled simulation data indicates crossover from Poisson statistics to stratification control at the $\beta_{\text {crit }}$ defined in Eq. (2), while the line passing close to the experimental data extrapolates to indicate crossover from $\Delta V^{2} \approx \Delta V_{\text {Poisson }}^{2}$ to stratification suppression at $\frac{1}{32} \beta_{\text {crit. }}$. We do not attempt further quantification here of the precise scalar multiple of the $\beta_{\text {crit }}$ defined in Eq. (2) at which crossover to stratification suppression of velocity fluctuations occurs, in light of the discovery that the crossover can shift so significantly between our simulations and experiments.

Various differences between the experiments and simulations could be responsible for this shift in the crossover stratification. The simulations include only point-force hydrodynamics, but that approximation should be reasonable at the low $\phi$ of the experiments. The simulated point-forces are additionally smoothed by the Fourier truncation, but results from different truncations do not appear to correlate in a noticeable way. Meanwhile, the procedure used to set up the initial stratified conditions in the experiments additionally introduced sucrose density gradients, yielding gradients in $\langle V\rangle$ and viscosity, while the simulations modeled the motion of initially stratified particles through a homogeneous suspending fluid. The dimensionless velocities considered eliminate any direct influence of different local viscosities; but it remains possible that the sucrose density gradients influ-

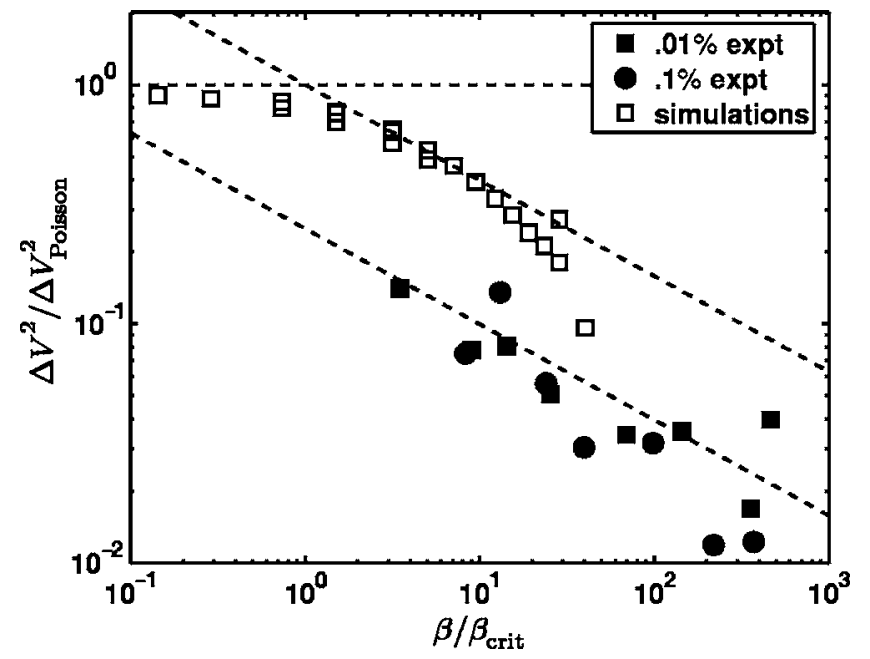

FIG. 4. Averaged vertical velocity variances (rescaled by the Poisson prediction) vs stratification. The experimental points are the same as those in the Fig. 2 inset. The simulation data points here are obtained from the data of Fig. 3, averaging each set of physical parameters over 5 simulation runs. The angled dashed lines identify the model-indicated exponent $-2 / 5$, with crossover stratifications at $\beta_{\text {crit }}$ and $\frac{1}{32} \beta_{\text {crit }}$, indicating that the crossover stratification above which stratification suppresses fluctuations in our experiments is $\approx 32$ times smaller than the critical stratification in our simulations.

enced the experiments in some unforeseen way. Finally, a major difference is geometrical; the experiments have a ratio of horizontal dimensions $w / d=1.5$, while the simulations are in cells with $w / d=4$ and 8 . Moreover the simulations are periodic in the long horizontal dimension, whereas the experiment is obviously influenced by the walls in the long horizontal dimension.

We hypothesize that the differences between the experimental and computational results here might be primarily driven by geometrical differences. Different geometries may also be relevant to understanding differences between various experiments. We note in particular that stratification control does not explain recent results with $h / d=2$ and square cross sections $(w=d),{ }^{11}$ nor with $h / d=4,{ }^{18}$ while Nguyen and Ladd $^{13}$ suggest (using simulations with $h / d$ large and square cross sections) that different controlling mechanisms may be at work depending on experimental details. It is particularly likely that the statistically steady arguments underlying the stratification suppression scaling break down without $h / d$ large, because the time scale of the initial swirl turnover becomes comparable to that of advection across the cell. Meanwhile, cross-sectional details might influence the precise crossover stratification because of differences in local mixing.

Ideally, the experimental and computational conditions would correspond more closely, but the simulation method used only provides a pair of no-slip side walls, and thus corresponds more closely to physical conditions with $w \gg d$ so as to minimize the influence of periodic boundary conditions in the long horizontal dimension, while the smaller $w / d$ ratio and the presence of the sucrose aided in setting up the initially imposed gradients in the experiments. In principle, future experiments could be conducted in cells with larger 
$w / d$ or further minimizing or even eliminating the sucrose density gradient.

Our initially stratified experiments and simulations are each consistent with the model for stratification suppression of velocity fluctuations, but with different constants setting the crossover stratifications. Other controlling mechanisms not studied here could of course prevail in other settings. Moreover, it is natural to question whether this scaling even makes sense at large stratifications where the suppressed correlation length becomes less than the interparticle scale. We note that the smallest swirl sizes deduced from $\ell / d \approx \Delta V^{2} / \Delta V_{\text {Poisson }}^{2}$ here correspond to a couple mean interparticle spacings in the simulations and are even below the mean spacings in the most stratified experiments. Meanwhile, we observe that the simulation data appears to indicate greater suppression of fluctuations beyond the model scaling at the largest stratifications considered. It remains possible that the $\Delta V^{2} / \Delta V_{\text {Poisson }}^{2} \sim\left(\beta / \beta_{\text {crit }}\right)^{-2 / 5}$ model needs modification for larger stratifications and cell-geometry effects, though it appears reasonably consistent with the experiments and simulations considered here.

This research was supported by NASA (NAG3-2376), the NSF (DMR-0602684, DMS-0204309, and DMS0605031), the Harvard MRSEC (DMR-0213805), and the DOE (DE-FG-02-03ER25567).

${ }^{1}$ W. O. Kermack, A. G. M'Kendrick, and E. Ponder, "The stability of suspensions. III. The velocities of sedimentation and of cataphoresis of suspensions in a viscous fluid," Proc. R. Soc. Edinburgh 49, 170 (1929).

${ }^{2}$ G. K. Batchelor, "Sedimentation in a dilute suspension of spheres," J. Fluid Mech. 52, 245 (1972).
${ }^{3}$ R. E. Caflisch and J. H. C. Luke, "Variance in the sedimentation speed of a suspension," Phys. Fluids 28, 759 (1985).

${ }^{4}$ J. M. Ham and G. M. Homsy, "Hindered settling and hydrodynamic dispersion in quiescent sedimenting suspensions," Int. J. Multiphase Flow 14, 533 (1988).

${ }^{5} \mathrm{H}$. Nicolai and E. Guazzelli, "Effect of the vessel size on the hydrodynamic diffusion of sedimenting spheres," Phys. Fluids 7, 3 (1995).

${ }^{6} \mathrm{P}$. N. Segrè, E. Herbolzheimer, and P. M. Chaikin, "Long-range correlations in sedimentation," Phys. Rev. Lett. 79, 2574 (1997).

${ }^{7}$ E. Guazzelli, "Evolution of particle-velocity correlations in sedimentation," Phys. Fluids 13, 1537 (2001).

${ }^{8}$ J. H. C. Luke, "Decay of velocity fluctuations in a stably stratified suspension," Phys. Fluids 12, 1619 (2000).

${ }^{9}$ S.-Y. Tee, P. J. Mucha, L. Cipelletti, S. Manley, M. P. Brenner, P. N. Segrè, and D. A. Weitz, "Nonuniversal velocity fluctuations of sedimenting particles," Phys. Rev. Lett. 89, 054501 (2002).

${ }^{10} \mathrm{P}$. J. Mucha and M. P. Brenner, "Diffusivities and front propagation in sedimentation," Phys. Fluids 15, 1305 (2003).

${ }^{11}$ L. Bergougnoux, S. Ghicini, E. Guazzelli, and J. Hinch, "Spreading fronts and fluctuations in sedimentation," Phys. Fluids 15, 1875 (2003).

${ }^{12}$ P. J. Mucha, S.-Y. Tee, D. A. Weitz, B. I. Shraiman, and M. P. Brenner, "A model for velocity fluctuations in sedimentation," J. Fluid Mech. 501, 71 (2004).

${ }^{13}$ N.-Q. Nguyen and A. J. C. Ladd, "Sedimentation of hard-sphere suspensions at low Reynolds number," J. Fluid Mech. 525, 73 (2005).

${ }^{14}$ D. Saintillan, E. S. G. Shaqfeh, and E. Darve, "The effect of stratification on the wave number selection in the instability of sedimenting spheroids," Phys. Fluids 18, 121503 (2006).

${ }^{15}$ E. J. Hinch, in Disorder and Mixing, edited by E. Guyon, J.-P. Nadal, and Y. Pomeau (Kluwer Academic, Dordrecht, 1988), Chap. IX, pp. 153-161.

${ }^{16}$ H. Nicolai, B. Herzhaft, E. J. Hinch, L. Oger, and E. Guazzelli, "Particle velocity fluctuations and hydrodynamic self-diffusion of sedimenting nonBrownian spheres," Phys. Fluids 7, 12 (1995).

${ }^{17}$ G. Oster, "Density gradients," Sci. Am. 213, 70 (1965).

${ }^{18}$ D. C. Gómez, L. Bergougnoux, J. Hinch, and E. Guazzelli, "On stratification control of the velocity fluctuations in sedimentation," Phys. Fluids 19, 098102 (2007). 